\title{
GLOBAL EXISTENCE AND ASYMPTOTIC BEHAVIOR OF WEAK SOLUTIONS \\ TO NONLINEAR THERMOVISCOELASTIC SYSTEMS WITH CLAMPED BOUNDARY CONDITIONS
}

\author{
$\mathrm{BY}$ \\ WEIXI SHEN (Department of Mathematics, Fudan University, Shanghai 200433, China), \\ SONGMU ZHENG (Institute of Mathematics, Fudan University, Shanghai 200433, China), \\ AND \\ PEICHENG ZHU (Institute of Mathematics, Fudan University, Shanghai 200433, China)
}

\begin{abstract}
This paper is concerned with global existence, uniqueness, and asymptotic behavior, as time tends to infinity, of weak solutions to nonlinear thermoviscoelastic systems with clamped boundary conditions. The constitutive assumptions for the Helmholtz free energy include the model for the study of phase transitions in shape memory alloys. To describe phase transitions between different configurations of crystal lattices, we work in a framework in which the strain $u$ belongs to $L^{\infty}$. It is shown that for any initial data of (strain, velocity, absolute temperature) $\left(u_{0}, v_{0}, \theta_{0}\right) \in$ $L^{\infty} \times W_{0}^{1, \infty} \times H^{1}$, there is a unique global solution $(u, v, \theta) \in C\left([0,+\infty] ; L^{\infty}\right) \times C(0,+\infty)$; $\left.W_{0}^{1, \infty}\right) \cap L^{\infty}\left([0,+\infty) ; W^{1, \infty}\right) \times C\left([0,+\infty) ; H^{1}\right)$. Results concerning the asymptotic behavior as time goes to infinity are obtained.
\end{abstract}

A new approach is introduced and more delicate estimates are derived to obtain the crucial $L^{\infty}$-norm estimate of $u$.

1. Introduction. This paper is concerned with global existence, uniqueness, and asymptotic behavior, as time tends to infinity, of weak solutions to the following system of one-dimensional nonlinear thermoviscoelasticity:

$$
\begin{gathered}
u_{t}-v_{x}=0 \\
v_{t}-\sigma_{x}=0 \\
\left(e+\frac{1}{2} v^{2}\right)_{t}-(\sigma v)_{x}+q_{x}=0 .
\end{gathered}
$$

Received April 1, 1996.

1991 Mathematics Subject Classification. Primary 35Q72, 73B30, 35B40.

The first author was supported by NSF of China, No. 19471016. The second author was supported by NSF of China, No. 19331040. 
Here $u, v, \sigma, e, \eta, \theta$, and $q$ denote deformation gradient (strain), velocity, stress, internal energy, specific entropy, absolute temperature, and heat flux, respectively. We assume that the reference configuration is the unit interval $[0,1]$.

For one-dimensional, homogeneous, thermoviscoelastic materials, internal energy, stress, entropy, and heat flux are given by the following constitutive relations:

$$
e=\hat{e}(u, \theta), \quad \sigma=\hat{\sigma}\left(u, \theta, v_{x}\right), \quad \eta=\hat{\eta}(u, \theta), \quad q=\hat{q}\left(u, \theta, \theta_{x}\right),
$$

which, in order to comply with the second law of thermodynamics expressed by the Clausius-Duhem inequality, must satisfy

$$
\begin{gathered}
\hat{\sigma}(u, \theta, 0)=\hat{\psi}_{u}(u, \theta), \quad \hat{\eta}(u, \theta)=-\hat{\psi}_{\theta}(u, \theta), \\
(\hat{\sigma}(u, \theta, w)-\hat{\sigma}(u, \theta, 0)) w \geq 0, \quad \hat{q}(u, \theta, g) g \leq 0
\end{gathered}
$$

where $\psi=e-\theta \eta$ is the Helmholtz free energy.

The following assumptions are made on our model:

(i) The material has viscoelastic damping of rate type, i.e.,

$$
\sigma=\frac{\partial \psi}{\partial u}+\gamma v_{x}
$$

with constant $\gamma>0$.

(ii) We assume that the heat flux $q$ is proportional to $\theta_{x}$ :

$$
q=-k \theta_{x}
$$

with constant $k>0$.

(iii) Concerning the Helmholtz free energy $\psi$, we assume that

$$
\psi(u, \theta)=-c_{v} \theta \ln \theta+c_{1} \theta+F_{1}(u) \theta+F_{2}(u),
$$

with positive constants $c_{v}, c_{1}$ and smooth functions $F_{1}, F_{2}$.

(iv) Concerning $F_{1}, F_{2}$, to include the model for the study of phase transition problems in shape memory alloys (see Falk [7], [8]), we assume that

$$
F_{1}(u)=u^{2}, \quad F_{2}(u)=u^{6}-c_{2} u^{4}-c_{3} u^{2}, \quad c_{2}, c_{3}>0 .
$$

However, more general models with similar properties of $F_{1}, F_{2}$ can also be treated. Thus the system (1.1)-(1.3) turns out to be

$$
\begin{gathered}
u_{t}-v_{x}=0 \\
v_{t}-\left(f_{1}(u) \theta+f_{2}(u)+\gamma v_{x}\right)_{x}=0, \\
c_{v} \theta_{t}-\theta f_{1}(u) v_{x}-\gamma v_{x}^{2}-k \theta_{x x}=0
\end{gathered}
$$

where $f_{1}=F_{1}^{\prime}, f_{2}=F_{2}^{\prime}$, and $\sigma$ is given by

$$
\sigma=f_{1}(u) \theta+f_{2}(u)+\gamma v_{x} .
$$

The equations (1.11)-(1.13) are supplemented by the following boundary conditions:

$$
\theta_{x}=0, \quad \text { for } x=0,1,
$$

and

$$
v=0, \quad \text { for } x=0,1,
$$


as well as by the initial conditions

$$
\left.u\right|_{t=0}=u_{0},\left.\quad v\right|_{t=0}=v_{0},\left.\quad \theta\right|_{t=0}=\theta_{0} .
$$

Boundary conditions (1.15)-(1.16) physically mean that there is no heat flux through the boundary and the rod is clamped at both ends.

To describe martensitic phase transitions between different configurations of crystal lattices, we have to work in a framework in which $u$ belongs to $L^{\infty}$. Therefore, we assume that

(v) $u_{0} \in L^{\infty}, v_{0} \in W_{0}^{1, \infty}, \theta_{0} \in H^{1}$ with $\theta_{0}>0$, for $x \in[0,1]$. Furthermore, the compatibility conditions $\left.v_{0}\right|_{x=0,1}=0$ are satisfied.

Before stating and proving our results, let us first recall the related results in the literature. Dafermos [5], Dafermos and Hsiao [6], and Jiang [11] proved the global existence of a classical solution to the system of (1.1)-(1.3) for a class of solid-like materials with stress-free boundary conditions at least at one end of the rod. The asymptotic behavior of smooth solutions as time tends to infinity has been investigated in Luo's thesis [12] for a special class of solid-like materials in which $e=c_{v} \theta$ and $F_{2} \equiv 0$. Recently, Racke and Zheng [16] investigated the global existence, uniqueness, and asymptotic behavior of weak solutions to the model in shape memory alloys also with a stress-free boundary condition at least at one end of the rod. In all of these papers, a variant of Andrews' technique used in Dafermos [5] and Pego [15] was crucial to obtain a uniform a priori estimate on the $L^{\infty}$-norm of $u$. However, this technique does not apply to the case where both ends of the rod are clamped. Thus the problem of global existence and uniqueness of classical solutions for the case in which both ends of the rod are clamped remained open for about ten years until the paper by Chen and Hoffmann [4]. Nevertheless, we should mention that in that paper the estimates on the solution crucially depend at least on the $H^{1}$-norm of initial data of $u$. As a result, their technique is not applicable to our problem with $u_{0}$ in $L^{\infty}$. In other words, to prove global existence of weak solutions, we have to adopt an entirely different approach. Besides, the estimates of the solutions that they obtained depended on $T$, an arbitrarily given length of time. Such estimates are enough to draw a conclusion on global existence, but not enough to study asymptotic behavior. Because of these two main difficulties, in this paper new techniques have to be introduced and more delicate estimates have to be derived (refer, e.g., to Lemma 2.2 and Lemma 2.3 to deal with our problem.

Concerning other references in this direction, for the model in shape memory alloys, we refer to Niezgódka and Sprekels [13] for the local existence of solutions in the weak sense. After that paper, uniqueness and global existence were proved by Hoffmann and Zheng [9], and by Niezgódka, Zheng, and Sprekels [14]. In the latter paper a viscous term for the temperature is introduced for the energy equation. We also refer to Sprekels and Zheng [18] and Hoffmann and Zochowski [10] for the global existence results for the model in shape memory alloys with the Helmholtz free energy density being a potential of Landau-Ginzburg form. Recently Sprekels, Zheng, and Zhu [19] obtained results on asymptotic behavior of solutions to the Laudau-Ginzburg model in shape memory alloys. We should also mention Andrews [2], Andrews and Ball [3], and Pego [15] for the purely viscoelastic case. 
The notation in this paper will be as follows: $L^{P}, 1 \leq p \leq \infty, W^{m, \infty}, m \in \mathbb{N}$, $H^{1} \equiv W^{1,2}, H_{0}^{1}=W_{0}^{1,2}$ denote the usual (Sobolev) spaces on $(0,1) ;\|\cdot\|_{B}$ denotes the norm in the space $B,\|\cdot\|:=\|\cdot\|_{L^{2}} ; C^{k}(I, B), k \in \mathbb{N}_{0}$ denotes the space of $k$-times continuously differentiable functions from $I \subset \mathbb{R}$ into a Banach space $B$; analogously: $L^{P}(I, B), 1 \leq p \leq \infty$; $\partial_{t}$ or $\frac{d}{d t}$ or a subscript $t$ and $\partial_{x}$ or a subscript $x$ denote the derivative with respect to $t$ and $x$ in the distribution sense, respectively.

We now define the weak solution to the problem (1.11)-(1.13) and (1.15)-(1.17):

Definition 1.1. For any $T>0$, we say that $(u, v, \theta)$ being in the set $(u, v, \theta) \in$ $C\left([0, T], L^{\infty}\right) \times C\left((0, T), W_{0}^{1, \infty}\right) \cap C\left([0, T], H_{0}^{1}\right) \cap L^{\infty}\left([0, T], W^{1, \infty}\right) \times C\left([0, T], H^{1}\right), u_{t} \in$ $C\left((0, T], L^{\infty}\right) \cap L^{\infty}\left([0, T], L^{\infty}\right), \theta_{x} \in L^{2}\left([0, T], H^{1}\right), v_{t}, \theta_{t} \in L^{2}\left([0, T], L^{2}\right)$ is a weak solution to the above initial boundary value problem if Eq. (1.1) is satisfied in $L^{\infty}\left([0, T], L^{\infty}\right)$, and for any $\varphi \in L^{2}\left([0, T], H_{0}^{1}\right), w \in L^{2}\left([0, T], H^{1}\right)$, the following hold:

$$
\begin{aligned}
& \int_{0}^{T} \int_{0}^{1} v_{t} \varphi d x d t+\int_{0}^{T} \int_{0}^{1} \sigma \varphi_{x} d x d t=0 \\
& \int_{0}^{T} \int_{0}^{1}\left(\left(c_{v} \theta_{t}-f_{1}(u) \theta v_{x}-\gamma v_{x}^{2}\right) w+k \theta_{x} w_{x}\right) d x d t=0
\end{aligned}
$$

and the initial conditions (1.17) are satisfied.

Now we can state our main theorem.

Theorem 1.2. Suppose that the assumptions (i)-(v) are satisfied. Then there exists a unique global weak solution $(u, v, \theta)$ defined above to the problem (1.11)-(1.13), (1.15)(1.17) with $\theta>0$ for all $(x, t) \in[0,1] \times[0, \infty)$. Moreover, as $t \rightarrow \infty$,

$$
\begin{gathered}
\|v(t)\|_{H^{1}} \rightarrow 0, \quad\left\|u_{t}(t)\right\| \rightarrow 0, \\
\left\|\theta_{x}(t)\right\| \rightarrow 0, \quad\|\theta(t)-\bar{\theta}(t)\|_{L^{\infty}} \rightarrow 0,
\end{gathered}
$$

where $\bar{\theta}(t):=\int_{0}^{1} \theta(x, t) d x$, and

$$
\left\|\sigma_{1}^{*}(t)\right\| \rightarrow 0, \quad\left\|\sigma^{*}(t)\right\| \rightarrow 0
$$

where

$$
\sigma_{1}=f_{1}(u) \theta+f_{2}(u), \quad \sigma_{1}^{*}=\sigma_{1}-\int_{0}^{1} \sigma_{1} d x, \quad \sigma^{*}=\sigma-\int_{0}^{1} \sigma d x .
$$

This paper is organized as follows. In section 2 we shall get uniform a priori estimates of solutions with smooth initial data. In section 3 we follow the idea by Andrews to obtain global existence and uniqueness of weak solutions. The results on asymptotic behavior are obtained in section 4 by combining the results in section 2 with a basic lemma in analysis by Shen and Zheng [17].

2. Uniform a priori estimates. In this section we assume that initial data $\left(u_{0}, v_{0}\right.$, $\left.\theta_{0}\right)$ are smooth functions. Therefore, as proved by Chen and Hoffmann [4], problem (1.11)-(1.13), (1.15)-(1.17) admits a unique global solution. In what follows we shall prove boundedness of

$$
\|u(t)\|_{L^{\infty}}, \quad\|v(t)\|_{W^{1, \infty}}, \quad\|\theta(t)\|_{H^{1}}
$$


uniformly in $t$. This will be done in a series of lemmata leading to the desired a priori estimates. Letters $C, C_{i}$ will denote universal constants not depending on $t$, but at most on $\left\|u_{0}\right\|_{L^{\infty}},\left\|v_{0}\right\|_{W^{1, \infty}}$, and $\left\|\theta_{0}\right\|_{H^{1}}$ of initial data. In the sequel, without loss of generality, we assume that $c_{v}=k=\gamma=1$.

LEMma 2.1. For any $t>0$, the following estimates hold:

$$
\begin{gathered}
\theta>0 \quad \text { in }[0,1] \times[0, \infty), \\
\|\theta(t)\|_{L^{1}}+\|v(t)\|^{2}+\|u\|_{L^{6}}^{6} \leq C, \\
\int_{0}^{t} \int_{0}^{1}\left(\frac{\theta_{x}^{2}}{\theta^{2}}+\frac{v_{x}^{2}}{\theta}\right) d x d \tau \leq C, \\
\int_{0}^{t}\|v(\tau)\|^{2} d \tau \leq \int_{0}^{t}\|v(\tau)\|_{L^{\infty}}^{2} d \tau \leq C, \\
0<C_{1 \alpha} \leq \int_{0}^{1} \theta(x, t)^{\alpha} d x \leq C_{2 \alpha}, \quad \forall \alpha \in(0,1] .
\end{gathered}
$$

Proof. First, applying the maximum principle to (1.13), we find that

$$
\theta(x, t)>0, \quad \forall(x, t) \in[0,1] \times \mathbb{R}^{+}
$$

Next, multiplying (1.12) by $v$, adding the result to (1.13) and integrating with respect to $x$ over $\Omega$, we arrive at

$$
\frac{d}{d t} \int_{0}^{1}\left(\theta+F_{2}(u)+\frac{1}{2} v^{2}\right) d x=0 .
$$

Hence,

$$
\int_{0}^{1}\left(\theta+F_{2}(u)+\frac{1}{2} v^{2}\right) d x=E_{1}
$$

where $E_{1}$ is a constant depending only on the initial data.

Using Young's inequality, we have

$$
F_{2}(u) \geq C_{1} u^{6}-C_{2}
$$

whence

$$
\|\theta(t)\|_{L^{1}}+\|v(t)\|^{2}+\|u\|_{L^{6}}^{6} \leq C .
$$

Multiplying (1.13) by $\theta^{-1}$ and integrating with respect to $x$ yields

$$
\frac{d}{d t}\left(\int_{0}^{1} \log \theta d x-\int_{0}^{1} F_{1}(u) d x\right)=\int_{0}^{1}\left(\frac{\theta_{x}^{2}}{\theta^{2}}+\frac{v_{x}^{2}}{\theta}\right) d x \geq 0 .
$$

Since $\log \theta \leq \theta-1$ for all $\theta>0$, we obtain

$$
\int_{0}^{t} \int_{0}^{1}\left(\frac{\theta_{x}^{2}}{\theta^{2}}+\frac{v_{x}^{2}}{\theta}\right) d x d \tau \leq C+C \int_{0}^{1}(\theta-1) d x \leq C .
$$

From $v(0, t)=0$ it follows that

$$
v(x, t)=v(0, t)+\int_{0}^{x} v_{y}(y, t) d y=\int_{0}^{x} v_{y}(y, t) d y .
$$


Thus,

$$
\begin{aligned}
\int_{0}^{t}\|v(\tau)\|_{L^{\infty}}^{2} d \tau & \leq \int_{0}^{t}\left(\int_{0}^{1} \sqrt{\theta} \frac{\left|v_{x}\right|}{\sqrt{\theta}} d x\right)^{2} d \tau \\
& \leq \int_{0}^{t}\left(\int_{0}^{1} \theta d x\right)\left(\int_{0}^{1} \frac{v_{x}^{2}}{\theta} d x\right) d \tau \\
& \leq C \int_{0}^{t} \int_{0}^{1} \frac{v_{x}^{2}}{\theta} d x d \tau \leq C
\end{aligned}
$$

Hence,

$$
\int_{0}^{t}\|v(\tau)\|^{2} d \tau \leq \int_{0}^{t}\|v(\tau)\|_{L^{\infty}}^{2} d \tau \leq C
$$

From (2.11) we have

$$
\int_{0}^{1} \log \theta d x-\int_{0}^{1} F_{1}(u) d x \geq \int_{0}^{1} \log \theta_{0} d x-\int_{0}^{1} F_{1}\left(u_{0}\right) d x .
$$

Therefore,

$$
\int_{0}^{1} \log \theta d x \geq-C_{4}
$$

and

$$
\alpha \int_{0}^{1} \log \theta d x=\int_{0}^{1} \log \theta^{\alpha} d x \geq-\alpha C_{4}, \quad \forall \alpha \in(0,1] .
$$

Applying Jensen's inequality, we find that

$$
\log \int_{0}^{1} \theta^{\alpha} d x \geq \int_{0}^{1} \log \theta^{\alpha} d x \geq-\alpha C_{4} .
$$

Therefore,

$$
\int_{0}^{1} \theta^{\alpha} d x \geq e^{-\alpha C_{4}}>0
$$

On the other hand, by Young's inequality,

$$
\int_{0}^{1} \theta^{\alpha} d x \leq \int_{0}^{1} \theta d x+C_{\alpha}, \quad \forall \alpha \in(0,1] .
$$

Combining (2.20) and (2.21), we get (2.5). Thus the proof is complete.

The following lemma plays an important role in estimating the $L^{\infty}$-norm of $u$.

LEMMA 2.2. $\forall \alpha \in(0,1]$ and for all $t>0$, the following estimates hold:

$$
\begin{gathered}
\int_{0}^{t} \int_{0}^{1}\left(\frac{\theta_{x}^{2}}{\theta^{1+\alpha}}+\frac{v_{x}^{2}}{\theta^{\alpha}}\right) d x d \tau \leq C_{\alpha} \\
\int_{0}^{t}\left\|\theta^{1-\alpha}-\int_{0}^{1} \theta^{1-\alpha} d x\right\|_{L^{\infty}}^{2} d \tau \leq C_{\alpha}, \quad \int_{0}^{t}\left\|v_{x}\right\|^{2} d \tau \leq C_{\alpha} \sup _{0 \leq \tau \leq t}\|\theta\|_{L^{\infty}}^{\alpha} .
\end{gathered}
$$


NONLINEAR THERMOVISCOELASTIC SYSTEMS

99

Proof. Suppose that (2.22) holds. Then

$$
\begin{gathered}
\int_{0}^{t}\left\|\theta^{1-\alpha}-\int_{0}^{1} \theta^{1-\alpha} d x\right\|_{L^{\infty}}^{2} d \tau \\
\leq C_{\alpha} \int_{0}^{t}\left|\int_{0}^{1} \frac{\theta_{x}}{\theta^{\alpha}} d x\right|^{2} d \tau \\
\leq C_{\alpha} \int_{0}^{t}\left(\int_{0}^{1} \frac{\theta_{x}^{2}}{\theta^{1+\alpha}} d x\right)\left(\int_{0}^{1} \theta^{1-\alpha} d x\right) d \tau \\
\leq C_{\alpha} \int_{0}^{t} \int_{0}^{1} \frac{\theta_{x}^{2}}{\theta^{1+\alpha}} d x d \tau \leq C_{\alpha} \\
\int_{0}^{t}\left\|v_{x}(\tau)\right\|^{2} d \tau \leq \int_{0}^{t}\left\|\theta^{\alpha / 2} \frac{v_{x}}{\theta^{\alpha / 2}}\right\|^{2} d \tau \\
\leq C_{\alpha} \sup _{0 \leq \tau \leq t}\|\theta\|_{L^{\infty}}^{\alpha} \int_{0}^{t} \int_{0}^{1} \frac{v_{x}^{2}}{\theta^{\alpha}} d x d \tau \\
\leq C_{\alpha} \sup _{0 \leq \tau \leq t}\|\theta\|_{L^{\infty}}^{\alpha} .
\end{gathered}
$$

Therefore, it remains to prove (2.22). We divide the proof into three steps.

First, let $\alpha \in\left[\frac{1}{2}, \frac{2}{3}\right]$. Multiplying (1.13) by $\theta^{-\alpha}\left(\int_{0}^{1} \theta^{1-\alpha} d x\right)^{-1}$ and integrating with respect to $x$ yields

$$
\begin{aligned}
0=\frac{1}{1-\alpha} & \left(\int_{0}^{1} \theta^{1-\alpha} d x\right)^{-1} \frac{d}{d t} \int_{0}^{1} \theta^{1-\alpha} d x \\
& \quad-\left(\int_{0}^{1} \theta^{1-\alpha} d x\right)^{-1}\left(\int_{0}^{1} f_{1} \theta^{1-\alpha} v_{x} d x+\int_{0}^{1}\left(\frac{\alpha \theta_{x}^{2}}{\theta^{1+\alpha}}+\frac{v_{x}^{2}}{\theta^{\alpha}}\right) d x\right) .
\end{aligned}
$$

However,

$$
\int_{0}^{1} f_{1} \theta^{1-\alpha} v_{x} d x=\int_{0}^{1} f_{1}\left(\theta^{1-\alpha}-\int_{0}^{1} \theta^{1-\alpha} d x\right) v_{x} d x+\int_{0}^{1} \theta^{1-\alpha} d x \frac{d}{d t} \int_{0}^{1} F_{1}(u) d x .
$$

Hence,

$$
\begin{aligned}
\left|\int_{0}^{t}\left(\int_{0}^{1} \theta^{1-\alpha} d x\right)^{-1} \int_{0}^{1} f_{1} \theta^{1-\alpha} v_{x} d x d \tau\right| \\
\leq\left|\int_{0}^{t}\left(\int_{0}^{1} \theta^{1-\alpha} d x\right)^{-1} \int_{0}^{1} f_{1}\left(\theta^{1-\alpha}-\int_{0}^{1} \theta^{1-\alpha} d x\right) v_{x} d x d \tau\right| \\
\quad+\left|\int_{0}^{t} \frac{d}{d t} \int_{0}^{1} F_{1} d x d \tau\right| \\
\leq C \int_{0}^{t}\left\|\theta^{1-\alpha}-\int_{0}^{1} \theta^{1-\alpha} d x\right\|_{L^{\infty}} \int_{0}^{1}|u| \theta^{\alpha / 2} \frac{\left|v_{x}\right|}{\theta^{\alpha / 2}} d x d \tau+C \\
\leq C \int_{0}^{t}\left\|\theta^{1-\alpha}-\int_{0}^{1} \theta^{1-\alpha} d x\right\|_{L^{\infty}}\left(\int_{0}^{1} u^{6} d x\right)^{\frac{1}{6}}\left(\int_{0}^{1} \theta^{\frac{3 \alpha}{2}} d x\right)^{\frac{1}{3}}\left(\int_{0}^{1} \frac{v_{x}^{2}}{\theta^{\alpha}} d x\right)^{\frac{1}{2}} d \tau+C .
\end{aligned}
$$


Since $\alpha \in\left[\frac{1}{2}, \frac{2}{3}\right]$, we have $\int_{0}^{1} \theta^{3 \alpha / 2} d x \leq C$. It follows from (2.28) and Lemma 2.1 that

$$
\begin{aligned}
& \left|\int_{0}^{t}\left(\int_{0}^{1} \theta^{1-\alpha} d x\right)^{-1} \int_{0}^{1} \theta^{1-\alpha} f_{1} v_{x} d x d \tau\right| \\
& \quad \leq C+C\left(\int_{0}^{t}\left\|\theta^{1-\alpha}-\int_{0}^{1} \theta^{1-\alpha} d x\right\|_{L^{\infty}}^{2} d \tau\right)^{1 / 2}\left(\int_{0}^{t} \int_{0}^{1} \frac{v_{x}^{2}}{\theta^{\alpha}} d x d \tau\right)^{1 / 2} \\
& \quad \leq C+\varepsilon \int_{0}^{t} \int_{0}^{1} \frac{v_{x}^{2}}{\theta^{\alpha}} d x d \tau+C \int_{0}^{t}\left(\int_{0}^{1} \frac{\left|\theta_{x}\right|}{\theta^{\alpha}} d x\right)^{2} d \tau \\
& \quad \leq C+\varepsilon \int_{0}^{t} \int_{0}^{1} \frac{v_{x}^{2}}{\theta^{\alpha}} d x d \tau+C \int_{0}^{t}\left(\int_{0}^{1} \frac{\theta_{x}^{2}}{\theta^{2}} d x \int_{0}^{1} \theta^{2(1-\alpha)} d x\right) d \tau
\end{aligned}
$$

with a small positive constant $\varepsilon$. Therefore, it follows from (2.26) that

$$
\int_{0}^{t} \int_{0}^{1}\left(\frac{\theta_{x}^{2}}{\theta^{1+\alpha}}+\frac{v_{x}^{2}}{\theta^{\alpha}}\right) d x d \tau \leq C, \quad \forall \alpha \in\left[\frac{1}{2}, \frac{2}{3}\right]
$$

Next, for $\alpha \in\left[\frac{1}{4}, \frac{1}{2}\right]$, in a similar manner we have

$$
\begin{aligned}
& \left|\int_{0}^{t}\left(\int_{0}^{1} \theta^{1-\alpha} d x\right)^{-1} \int_{0}^{1} f_{1} \theta^{1-\alpha} v_{x} d x d \tau\right| \\
& \quad \leq C+C\left(\int_{0}^{t}\left\|\theta^{1-\alpha}-\int_{0}^{1} \theta^{1-\alpha} d x\right\|_{L^{\infty}}^{2} d \tau\right)^{1 / 2}\left(\int_{0}^{t} \int_{0}^{1} \frac{v_{x}^{2}}{\theta^{\alpha}} d x d \tau\right)^{1 / 2} \\
& \quad \leq C+\varepsilon \int_{0}^{t} \int_{0}^{1} \frac{v_{x}^{2}}{\theta^{\alpha}} d x d \tau+\int_{0}^{t} \int_{0}^{1} \frac{\theta_{x}^{2}}{\theta^{3 / 2}} d x \int_{0}^{1} \theta^{\frac{3}{2}-2 \alpha} d x d \tau .
\end{aligned}
$$

Thus we deduce from (2.30), (2.26), and (2.31) that

$$
\int_{0}^{t} \int_{0}^{1}\left(\frac{\theta_{x}^{2}}{\theta^{1+\alpha}}+\frac{v_{x}^{2}}{\theta^{\alpha}}\right) d x d \tau \leq C, \quad \forall \alpha \in\left[\frac{1}{4}, \frac{1}{2}\right]
$$

A simple induction argument yields that for any $n \in N$, the following inequality holds:

$$
\int_{0}^{t} \int_{0}^{1}\left(\frac{\theta_{x}^{2}}{\theta^{1+\alpha}}+\frac{v_{x}^{2}}{\theta^{\alpha}}\right) d x d \tau \leq C, \quad \forall \alpha \in\left[\frac{1}{2^{n+1}}, \frac{1}{2^{n}}\right]
$$

Combining (2.33) with (2.30), we conclude that (2.22) is valid for any $\alpha \in\left(0, \frac{2}{3}\right]$. 
Finally, we show that (2.22) holds for any $\alpha \in\left(\frac{2}{3}, 1\right]$. Indeed, for $\beta \in\left(0, \frac{2}{3}\right]$ we have

$$
\begin{aligned}
& \left|\int_{0}^{t}\left(\int_{0}^{1} \theta^{1-\alpha} d x\right)^{-1} \int_{0}^{1} f_{1} \theta^{1-\alpha} v_{x} d x d \tau\right| \\
& \quad \leq C+C \int_{0}^{t}\left\|\theta^{1-\alpha}-\int_{0}^{1} \theta^{1-\alpha} d x\right\|_{L^{\infty}} \int_{0}^{1}|u| \theta^{\beta / 2} \frac{\left|v_{x}\right|}{\theta^{\beta / 2}} d x d \tau \\
& \quad \leq C+C \int_{0}^{t}\left\|\theta^{1-\alpha}-\int_{0}^{1} \theta^{1-\alpha} d x\right\|_{L^{\infty}}\left(\int_{0}^{1} u^{6} d x\right)^{\frac{1}{6}}\left(\int_{0}^{1} \theta^{\frac{3 \beta}{2}} d x\right)^{\frac{1}{3}}\left(\int_{0}^{1} \frac{v_{x}^{2}}{\theta^{\beta}} d x\right)^{\frac{1}{2}} d \tau \\
& \quad \leq C+C\left(\int_{0}^{t}\left(\int_{0}^{1} \frac{\left|\theta_{x}\right|}{\theta^{\alpha}} d x\right)^{2} d \tau\right)^{\frac{1}{2}}\left(\int_{0}^{1} \int_{0}^{\frac{1}{2}} \frac{v_{x}^{2}}{\theta^{\beta}} d x d \tau\right)^{\frac{1}{2}} \\
& \quad \leq C+C\left(\int_{0}^{t} \frac{\theta_{x}^{2}}{\theta^{2}} d x \int_{0}^{1} \theta^{2(1-\alpha)} d x d \tau\right)^{1} \\
& \quad \leq C .
\end{aligned}
$$

Therefore, we infer from (2.34), (2.33), and (2.30) that

$$
\int_{0}^{t} \int_{0}^{1}\left(\frac{\theta_{x}^{2}}{\theta^{1+\alpha}}+\frac{v_{x}^{2}}{\theta^{\alpha}}\right) d x d \tau \leq C, \quad \forall \alpha \in(0,1] .
$$

Thus, the proof is complete.

The following lemma gives a uniform a priori estimate on the $L^{\infty}$-norm of $u$ and will play a crucial role in this paper.

Lemma 2.3. For any $T>0$, there exists a positive constant $C$ depending only on the norm of $u_{0}, v_{0}, \theta_{0}$ in $L^{\infty} \times W^{1, \infty} \times H^{1}$, but independent of $T$ such that

$$
\|u\|_{L^{\infty}([0,1] \times[0, T])} \leq C .
$$

Proof. Let

$$
G(x, y, t)=\sum_{n=1}^{\infty} e^{-(n \pi)^{2} t} \sin n \pi x \sin n \pi y
$$

which is the fundamental solution to the heat equation subject to the Dirichlet boundary condition. Then Eq. (1.12) can be rewritten as

$$
v_{t}-v_{x x}-\sigma_{1 x}^{*}=0
$$

with

$$
\sigma_{1}^{*}=\sigma_{1}-\int_{0}^{1} \sigma_{1} d x
$$

Therefore, $v$ satisfies the following integral equation:

$$
v(x, t)=\int_{0}^{1} G(x, y, t) v_{0}(y) d y-\int_{0}^{t} \int_{0}^{1} G_{y}(x, y, t-s) \sigma_{1}^{*}(y, s) d y d s .
$$


Let

$$
H(x, y, t)=\sum_{n=0}^{\infty} e^{-(n \pi)^{2} t} \cos n \pi x \cos n \pi y,
$$

which is the fundamental solution to the heat equation subject to the Neumann boundary condition with the property $G_{y}=-H_{x}$. Thus, by integration by parts, we have

$$
\begin{aligned}
v_{x} & =\int_{0}^{1} G_{x}(x, y, t) v_{0}(y) d y-\frac{\partial}{\partial x} \int_{0}^{t} \int_{0}^{1} G_{y}(x, y, t-s) \sigma_{1}^{*}(y, s) d y d s \\
& =\int_{0}^{1} H(x, y, t) v_{0}^{\prime}(y) d y+\frac{\partial^{2}}{\partial x^{2}} \int_{0}^{t} \int_{0}^{1} H(x, y, t-s) \sigma_{1}^{*}(y, s) d y d s \\
& =\int_{0}^{1} H(x, y, t) v_{0}^{\prime} d y+\frac{\partial}{\partial t} \int_{0}^{t} \int_{0}^{1} H(x, y, t-s) \sigma_{1}^{*}(y, s) d y d s-\sigma_{1}^{*}(x, t) .
\end{aligned}
$$

Since $v_{0} \in W^{1, \infty}$, as proved by Andrews [2], for all $t>0$,

$$
\left|\int_{0}^{1} H(x, y, t) v_{0}^{\prime}(y) d y\right| \leq C \text {. }
$$

In what follows we prove that for all $(x, t) \in[0,1] \times \mathbb{R}^{+}$,

$$
\left|\int_{0}^{t} \int_{0}^{1} H(x, y, t-s) \sigma_{1}^{*}(y, s) d y d s\right| \leq C .
$$

To this end, we write

$$
\sigma_{1}^{*}=f(x, t)+g(x, t)
$$

with

$$
f(x, t)=f_{1}(u)\left(\theta-\left(\int_{0}^{1} \theta^{\frac{3}{4}} d x\right)^{\frac{4}{3}}\right)-\int_{0}^{1} f_{1}(u)\left(\theta-\left(\int_{0}^{1} \theta^{\frac{3}{4}} d x\right)^{\frac{4}{3}}\right) d x
$$

and

$$
g(x, t)=f_{2}-\int_{0}^{1} f_{2} d x+\left(f_{1}-\int_{0}^{1} f_{1} d x\right)\left(\int_{0}^{1} \theta^{\frac{3}{4}} d x\right)^{\frac{4}{3}}
$$

Clearly,

$$
g \in L^{\infty}\left(0, \infty ; L^{\frac{6}{5}}\right)
$$

Thus,

$$
\begin{array}{r}
\left|\int_{0}^{t} \int_{0}^{1} H(\cdot, y, t-s) g(y, s) d y d s\right|_{L^{\infty}} \\
\quad \leq C \int_{0}^{t} \sum_{n=1}^{\infty} e^{-(n \pi)^{2}(t-s)}\|g\|_{L^{1}} d s \\
\quad \leq C \int_{0}^{t} \sum_{n=1}^{\infty} e^{-(n \pi)^{2}(t-s)} d s \leq C .
\end{array}
$$


Let

$$
w=\int_{0}^{t} \int_{0}^{1} H(x, y, t-s) f(y, s) d y d s
$$

Then $w$ satisfies

$$
\begin{gathered}
w_{t}-w_{x x}=f \\
\left.w_{x}\right|_{x=0,1}=0, \quad w(x, 0)=0 .
\end{gathered}
$$

It is easy to see that

$$
\int_{0}^{1} w(x, t) d x=\int_{0}^{1} w(x, 0) d x=0
$$

Hence,

$$
\|w\|_{L^{\infty}} \leq C\left\|w_{x}\right\|
$$

Multiplying (2.51) by $w_{t}$ and integrating with respect to $x$, we get

$$
\begin{aligned}
& \frac{1}{2} \frac{d}{d t}\left\|w_{x}\right\|^{2}+\left\|w_{t}\right\|^{2}=\left(f, w_{t}\right) \\
& \leq \frac{1}{2}\left\|w_{t}\right\|^{2}+C \int_{0}^{1} u^{2}\left(\theta-\left(\int_{0}^{1} \theta^{\frac{3}{4}} d x\right)^{\frac{4}{3}}\right)^{2} d x \\
& \leq \frac{1}{2}\left\|w_{t}\right\|^{2}+C \int_{0}^{1} u^{2}\left|\theta^{\frac{3}{4}}-\int_{0}^{1} \theta^{\frac{3}{4}} d x\right|^{2}\left|\theta^{\frac{1}{3}}+\left(\int_{0}^{1} \theta^{\frac{3}{4}} d x\right)^{\frac{1}{3}}\right|^{2} d x \\
& \leq \frac{1}{2}\left\|w_{t}\right\|^{2}+C\left\|\theta^{\frac{3}{4}}-\int_{0}^{1} \theta^{\frac{3}{4}} d x\right\|_{L^{\infty}}^{2}\left(\int_{0}^{1} u^{6} d x\right)^{\frac{1}{3}}\left(\int_{0}^{1}\left(\theta^{\frac{1}{3}}+C\right)^{3} d x\right)^{\frac{2}{3}} \\
& \leq \frac{1}{2}\left\|w_{t}\right\|^{2}+C\left\|\theta^{\frac{3}{4}}-\int_{0}^{1} \theta^{\frac{3}{4}} d x\right\|_{L^{\infty}} \cdot
\end{aligned}
$$

Hence, using (2.23) with $\alpha=1 / 4$, we get

$$
\left\|w_{x}\right\|^{2}+\int_{0}^{t}\left\|w_{t}\right\|^{2} d \tau \leq C \int_{0}^{t}\left\|\theta^{\frac{3}{4}}-\int_{0}^{1} \theta^{\frac{3}{4}} d x\right\|_{L^{\infty}}^{2} d \tau \leq C .
$$

Thus it follows from (2.54) that

$$
\|w\|_{L^{\infty}} \leq C
$$

i.e.,

$$
\left|\int_{0}^{t} \int_{0}^{1} \dot{H}(\cdot, y, t-s) f(y, s) d y d s\right|_{L^{\infty}} \leq C .
$$

We deduce from (2.58), (2.49), and (2.45) that

$$
\left|\int_{0}^{t} \int_{0}^{1} H(\cdot, y, t-s) \sigma_{1}^{*}(y, s) d y d s\right|_{L^{\infty}} \leq C .
$$

For any $T>0$, let

$$
M=M(T)=\sup _{0 \leq x \leq 1,0 \leq t \leq T}|u(x, t)| .
$$


In what follows we prove that $M$ is uniformly bounded by a positive constant depending only on the norm of $u_{0}, v_{0}, \theta_{0}$ in $L^{\infty} \times W^{1, \infty} \times H^{1}$.

Let

$$
q(x, t)=u(x, t)-\int_{0}^{t} \int_{0}^{1} H(x, y, t-s) \sigma_{1}^{*}(y, s) d y d s .
$$

Then by (1.11), (2.42), and (2.61) we have

$$
\frac{\partial q}{\partial t}(x, t)=-\sigma_{1}^{*}(x, t)+\int_{0}^{1} H(x, y, t) v_{0}^{\prime} d y .
$$

Since $u_{0} \in L^{\infty}([0,1])$, we have

$$
|q(x, 0)|=|u(x, 0)| \leq M_{0} \leq M^{1 / 2}+M_{0}
$$

where $M_{0}=\left\|u_{0}\right\|_{L^{\infty}}$.

We now use the contradiction argument. Suppose now that for some $x_{0} \in[0,1]$ there exists $t_{0} \in[0, T]$ such that

$$
q\left(x_{0}, t_{0}\right)>M^{1 / 2}+M_{0}
$$

Recall that because initial data are assumed to be smooth in this section, by the result by Chen and Hoffmann [4], the solution is smooth. Therefore, the mapping $t \rightarrow q\left(x_{0}, t\right)$, for this fixed $x_{0} \in[0,1]$, is continuously differentiable. Thus this implies that there exists $t^{*} \in\left(0, t_{0}\right)$ such that

$$
\begin{gathered}
q\left(x_{0}, t^{*}\right)=M^{1 / 2}+M_{0}, \\
q\left(x_{0}, t\right) \leq M^{1 / 2}+M_{0} \quad \text { for } t \in\left[0, t^{*}\right],
\end{gathered}
$$

and

$$
\frac{\partial q}{\partial t}\left(x_{0}, t^{*}\right) \geq 0
$$

Now (2.61), (2.65), and (2.44) imply that

$$
u\left(x_{0}, t^{*}\right) \geq M^{1 / 2}+M_{0}-C .
$$

Without loss of generality, we can assume that the term on the right-hand side of (2.68) is positive. Otherwise, the proof is done.

Using Young's inequality we get

$$
f_{2}(u) \geq \frac{1}{2} u^{5}-C, \quad \forall u>0 .
$$

It is easy to see that

$$
\left|\int_{0}^{1} u \theta d x\right| \leq M \int_{0}^{1} \theta d x \leq C M
$$

Since $\theta>0$, we have

$$
\left.\left(\sigma_{1}^{*}-\int_{0}^{1} H(x, y, t) v_{0}^{\prime} d y\right)\right|_{\left(x_{0}, t^{*}\right)} \geq \frac{1}{2}\left(M^{1 / 2}+M_{0}-C\right)^{5}-C-C M .
$$


Therefore, there exists a positive constant $M_{1}$ depending only on $M_{0}$ and $C$, i.e., the norm of $u_{0}, v_{0}, \theta_{0}$ in $L^{\infty} \times W^{1, \infty} \times H^{1}$ such that if $M>M_{1}$, then

$$
\left.\left(\sigma_{1}^{*}-\int_{0}^{1} H(x, y, t) v_{0}^{\prime} d y\right)\right|_{\left(x_{0}, t^{*}\right)}>0 .
$$

Combining this with (2.62) yields

$$
\frac{\partial q}{\partial t}\left(x_{0}, t^{*}\right)<0
$$

which contradicts the inequality (2.67). We conclude from the above argument that either $M$ is uniformly bounded or for all $(x, t)$,

$$
q(x, t) \leq M^{1 / 2}+M_{0} .
$$

In the same manner, we can prove that either $M$ is uniformly bounded or for all $(x, t)$,

$$
q(x, t) \geq-\left(M^{1 / 2}+M_{0}\right) .
$$

Hence, we conclude that either $M$ is uniformly bounded or for all $(x, t)$,

$$
|q(x, t)| \leq M^{1 / 2}+M_{0}
$$

We now show that if (2.76) holds, then $M$ is also uniformly bounded. Indeed, by (2.76), (2.61), and (2.44), we obtain

$$
|u(x, t)| \leq M^{1 / 2}+M_{0}+C, \quad \forall(x, t) \in[0,1] \times[0, T] .
$$

Thus by the definition of $M$,

$$
M \leq M^{1 / 2}+M_{0}+C \leq \frac{M}{2}+\frac{1}{2}+M_{0}+C
$$

whence

$$
M \leq 1+2 M_{0}+2 C
$$

Thus the proof is complete.

Lemma 2.4. For all $t>0$ and any $\alpha>0$, the following estimates hold:

$$
\begin{aligned}
\left\|v_{x}\right\|^{2} & +\int_{0}^{t}\left\|v_{t}\right\|^{2} d \tau \\
& \leq C_{\alpha}\left(1+\sup _{0 \leq \tau \leq t}\|\theta\|_{L^{\infty}}^{1+\alpha}+\sup _{0 \leq \tau \leq t}\|\theta\|_{L^{\infty}}^{\alpha / 2} \sqrt{\int_{0}^{t}\left\|\theta_{t}\right\|^{2} d \tau}\right), \\
& \int_{0}^{t}\left\|\sigma_{1}^{*}\right\|^{2} d \tau \leq C_{\alpha}\left(1+\sup _{0 \leq \tau \leq t}\|\theta\|_{L^{\infty}}^{1+\alpha}+\sqrt{\int_{0}^{t}\left\|\theta_{t}\right\|^{2} d \tau}\right) .
\end{aligned}
$$

Proof. Multiplying (2.38) by $-\int_{0}^{x} \sigma_{1}^{*} d x$ and integrating with respect to $x$ yields

$$
\left\|\sigma_{1}^{*}\right\|^{2}=\left(-\int_{0}^{x} \sigma_{1}^{*} d x, \sigma_{1 x}^{*}\right)=\left(v_{t}-v_{x x},-\int_{0}^{x} \sigma_{1}^{*} d x\right) .
$$


By integration by parts, we easily get

$$
\begin{aligned}
\left(v_{t},-\int_{0}^{x} \sigma_{1}^{*} d x\right) & =\left(\frac{\partial}{\partial x} \int_{0}^{x} v_{t} d x,-\int_{0}^{x} \sigma_{1}^{*} d x\right) \\
& =\left(\int_{0}^{x} v_{t} d x, \sigma_{1}^{*}\right)=\frac{\partial}{\partial t}\left(\int_{0}^{x} v d x, \sigma_{1}^{*}\right)-\left(\int_{0}^{x} v d x, \sigma_{1 t}^{*}\right)
\end{aligned}
$$

where

$$
\sigma_{1 t}=f_{1} \theta_{t}+f_{1}^{\prime} \theta v_{x}+f_{2}^{\prime} v_{x} .
$$

Hence, by Lemma 2.1-Lemma 2.3, for any $\alpha \in(0,1]$ we have

$$
\begin{aligned}
& \left|\int_{0}^{t}\left(v_{t},-\int_{0}^{x} \sigma_{1}^{*} d x\right) d \tau\right| \leq C+\left|\left(\int_{0}^{x} v d x, \sigma_{1}^{*}\right)\right|+\left|\int_{0}^{t}\left(\int_{0}^{x} v d x, \sigma_{1 t}^{*}\right) d \tau\right| \\
& \quad \leq C+C\|v\|_{L^{1}}\left\|\sigma_{1}^{*}\right\|_{L^{1}}+C \int_{0}^{t}\|v\|_{L^{1}}\left\|\sigma_{1 t}^{*}\right\|_{L^{1}} d \tau \\
& \quad \leq C+C\left(\int_{0}^{t}\|v\|_{L^{1}}^{2} d \tau\right)^{1 / 2}\left(\int_{0}^{t}\left\|\sigma_{1 t}^{*}\right\|_{L^{1}}^{2} d \tau\right)^{1 / 2} \\
& \quad \leq C+C\left(\int_{0}^{t}\left(\left\|\theta_{t}\right\|^{2}+\left\|\theta v_{x}\right\|_{L^{1}}^{2}+\left\|v_{x}\right\|^{2}\right) d \tau\right)^{1 / 2} \\
& \quad \leq C\left(1+\sup _{0 \leq \tau \leq t}\|\theta\|_{L^{\infty}}^{1+\alpha}+\sup _{0 \leq \tau \leq t}\|\theta\|_{L^{\infty}}^{\alpha / 2}+\sqrt{\int_{0}^{t}\left\|\theta_{t}\right\|^{2} d \tau}\right) \\
& \quad \leq C\left(1+\sup _{0 \leq \tau \leq t}\|\theta\|_{L^{\infty}}^{1+\alpha}+\sqrt{\int_{0}^{t}\left\|\theta_{t}\right\|^{2} d \tau}\right) .
\end{aligned}
$$

On the other hand, by integration by parts and Lemma 2.2 we have

$$
\begin{aligned}
\left|\int_{0}^{t}\left(v_{x x}, \int_{0}^{x} \sigma_{1}^{*}\right) d \tau\right| & \leq\left|\int_{0}^{t}\left(v_{x}, \sigma_{1}^{*}\right) d \tau\right| \\
& \leq \frac{1}{2} \int_{0}^{t}\left\|\sigma_{1}^{*}\right\|^{2} d \tau+C \int_{0}^{t}\left\|v_{x}\right\|^{2} d \tau \\
& \leq \frac{1}{2} \int_{0}^{t}\left\|\sigma_{1}^{*}\right\|^{2} d \tau+C \sup _{0 \leq \tau \leq t}\|\theta\|_{L^{\infty}}^{\alpha} .
\end{aligned}
$$

Combining (2.82) with (2.85) and (2.86), we obtain

$$
\int_{0}^{t}\left\|\sigma_{1}^{*}\right\|^{2} d \tau \leq C\left(1+\sup _{0 \leq \tau \leq t}\|\theta\|_{L^{\infty}}^{1+\alpha}+\sqrt{\int_{0}^{t}\left\|\theta_{t}\right\|^{2} d \tau}\right) .
$$

Now we multiply $(2.38)$ by $v_{t}$ and integrate with respect to $x$ to get

$$
\begin{aligned}
0 & =\frac{1}{2} \frac{d}{d t}\left\|v_{x}\right\|^{2}+\left\|v_{t}\right\|^{2}-\int_{0}^{1} \sigma_{1 x}^{*} v_{t} d x \\
& =\frac{1}{2} \frac{d}{d t}\left\|v_{x}\right\|^{2}+\left\|v_{t}\right\|^{2}+\int_{0}^{1} \sigma_{1}^{*} v_{x t} d x
\end{aligned}
$$


Since

$$
\int_{0}^{1} \sigma_{1}^{*} v_{x t} d x=\frac{d}{d t} \int_{0}^{1} \sigma_{1}^{*} v_{x} d x-\int_{0}^{1} \sigma_{1 t}^{*} v_{x} d x
$$

we deduce that

$$
\begin{aligned}
& \left|\int_{0}^{t} \int_{0}^{1} \sigma_{1}^{*} v_{x t} d x d \tau\right| \\
& \quad \leq C+\left|\int_{0}^{1} \sigma_{1}^{*} v_{x} d x\right|+\left(\int_{0}^{t}\left\|v_{x}\right\|^{2} d \tau\right)^{1 / 2}\left(\int_{0}^{t}\left\|\sigma_{1 t}^{*}\right\|^{2} d \tau\right)^{1 / 2} \\
& \quad \leq C+\frac{1}{4}\left\|v_{x}\right\|^{2}+C\left\|\sigma_{1}^{*}\right\|^{2}+C \sup _{0 \leq \tau \leq t}\|\theta\|_{L^{\infty}}^{\alpha / 2}\left(\int_{0}^{t}\left\|\theta_{t}\right\|^{2}+\left\|v_{x}\right\|^{2}+\left\|\theta v_{x}\right\|^{2} d \tau\right)^{1 / 2} \\
& \quad \leq C+\frac{1}{4}\left\|v_{x}\right\|^{2}+C \sup _{0 \leq \tau \leq t}\|\theta\|_{L^{\infty}} \\
& \quad+C \sup _{0 \leq \tau \leq t}\|\theta\|_{L^{\infty}}^{\alpha / 2}\left(\sup _{0 \leq \tau \leq t}\|\theta\|_{L^{\infty}}^{1+\alpha}+\sup _{0 \leq \tau \leq t}\|\theta\|_{L^{\infty}}^{\alpha / 2}+\sqrt{\int_{0}^{t}\left\|\theta_{t}\right\|^{2} d \tau}\right) .
\end{aligned}
$$

Combining this with (2.88) yields

$$
\left\|v_{x}\right\|^{2}+\int_{0}^{t}\left\|v_{t}\right\|^{2} d \tau \leq C\left(1+\sup _{0 \leq \tau \leq t}\|\theta\|_{L^{\infty}}^{1+\alpha}+\sup _{0 \leq \tau \leq t}\|\theta\|_{L^{\infty}}^{\alpha / 2} \sqrt{\int_{0}^{t}\left\|\theta_{t}\right\|^{2} d \tau}\right) .
$$

Thus, the proof is complete.

Next we get uniform a priori estimates on $\left\|v_{x}\right\|_{L^{\infty}}$.

LEMMA 2.5. For all $t>0$, we have

$$
\sup _{0 \leq \tau \leq t}\left\|v_{x}\right\|_{L^{\infty}} \leq C\left(1+\sup _{0 \leq \tau \leq t}\|\theta\|_{L^{\infty}}+\left(\int_{0}^{t}\left\|\theta_{\tau}\right\|^{2} d \tau\right)^{7 / 16}\right) .
$$

Proof. It follows from (2.42) that

$$
\begin{aligned}
v_{x}= & \int_{0}^{1} H(x, y, t) v_{0}^{\prime}(y) d y-\sigma_{1}^{*}+\frac{\partial}{\partial t} \int_{0}^{t} \int_{0}^{1} H(x, y, t-s) \sigma_{1}^{*}(y, s) d y d s \\
= & \int_{0}^{1} H(x, y, t) v_{0}^{\prime}(y) d y-\sigma_{1}^{*}+\int_{0}^{1} H(x, y, t) \sigma_{1}^{*}(y, 0) d y \\
& \quad+\int_{0}^{t} \int_{0}^{1} H(x, y, t) \sigma_{1 s}^{*}(y, s) d y d s .
\end{aligned}
$$

Let

$$
\zeta(x, t)=\int_{0}^{t} \int_{0}^{1} H(x, y, t-s) \sigma_{1 s}^{*}(y, s) d y d s
$$

Then $\zeta$ satisfies

$$
\begin{gathered}
\zeta_{t}-\zeta_{x x}=\left(\sigma_{1}^{*}\right)_{t}, \\
\left.\zeta_{x}\right|_{x=0,1}=0, \quad \zeta(x, 0)=0 .
\end{gathered}
$$


It is easy to see that

$$
\int_{0}^{1} \zeta(x, t) d x=\int_{0}^{1} \zeta(x, 0) d x=0
$$

Thus

$$
\|\zeta\| \leq C\left\|\zeta_{x}\right\|, \quad\|\zeta\|_{L^{\infty}} \leq C\left\|\zeta_{x}\right\| .
$$

We now multiply $(2.95)$ by $\zeta_{t}$ and integrate with respect to $x$ to get

$$
\frac{1}{2} \frac{d}{d t}\left\|\zeta_{x}\right\|^{2}+\left\|\zeta_{t}\right\|^{2} \leq \frac{1}{2}\left\|\zeta_{t}\right\|^{2}+C\left(\left\|v_{x}\right\|^{2}+\|\theta\|_{L^{\infty}}^{2}\left\|v_{x}\right\|^{2}+\left\|\theta_{t}\right\|^{2}\right) .
$$

Thus, for any $\alpha>0$

$$
\left\|\zeta_{x}\right\|^{2}+\int_{0}^{t}\left\|\zeta_{t}\right\|^{2} d \tau \leq C\left(1+\max _{0 \leq \tau \leq t}\|\theta\|_{L^{\infty}}^{2+\alpha}+\int_{0}^{t}\left\|\theta_{t}\right\|^{2} d \tau\right) .
$$

On the other hand, we easily get

$$
\frac{1}{2} \frac{d}{d t}\|\zeta\|^{2}+\left\|\zeta_{x}\right\|^{2}=\int_{0}^{1} \zeta\left(\sigma_{1}^{*}\right)_{t} d x=\frac{d}{d t} \int_{0}^{1} \zeta \sigma_{1}^{*} d x-\int_{0}^{1} \zeta_{t} \sigma_{1}^{*} d x
$$

Therefore,

$$
\begin{aligned}
\frac{1}{2}\|\zeta\|^{2} & -\int_{0}^{1} \zeta \sigma_{1}^{*}+\int_{0}^{t}\left\|\zeta_{x}\right\|^{2} d \tau \\
& \leq \int_{0}^{t}\left\|\zeta_{t}\right\|\left\|\sigma_{1}^{*}\right\| d \tau \\
& \leq\left(\int_{0}^{t}\left\|\sigma_{1}^{*}\right\|^{2} d \tau\right)^{1 / 2}\left(\int_{0}^{t}\left\|\zeta_{t}\right\|^{2} d \tau\right)^{1 / 2} \\
& \leq C\left(1+\sup _{0 \leq \tau \leq t}\|\theta\|_{L^{\infty}}^{1+\alpha}+\sqrt{\int_{0}^{t}\left\|\theta_{\tau}\right\|^{2} d \tau}\right)^{1 / 2}\left(\int_{0}^{t}\left\|\zeta_{t}\right\|^{2} d \tau\right)^{1 / 2} .
\end{aligned}
$$

Since we also have $\left\|\sigma_{1}^{*}\right\|^{2} \leq C+C \sup _{0 \leq \tau \leq t}\|\theta\|_{L^{\infty}}$, we obtain

$$
\begin{aligned}
\|\zeta(t)\|^{2} & \leq C\left(1+\sup _{0 \leq \tau \leq t}\|\theta\|_{L^{\infty}}+\left(1+\sup _{0 \leq \tau \leq t}\|\theta\|_{L^{\infty}}^{2+\alpha}+\int_{0}^{t}\left\|\theta_{t}\right\|^{2} d \tau\right)^{3 / 4}\right) \\
& \leq C\left(1+\sup _{0 \leq \tau \leq t}\|\theta\|_{L^{\infty}}^{2+\alpha}+\int_{0}^{t}\left\|\theta_{t}\right\|^{2} d \tau\right)^{3 / 4} .
\end{aligned}
$$

Hence by Young's inequality we can easily get

$$
\begin{aligned}
\|\zeta\|_{L^{\infty}}^{2} & \leq C\left\|\zeta_{x}\right\|\|\zeta\| \\
& \leq C\left(1+\sup _{0 \leq \tau \leq t}\|\theta\|_{L^{\infty}}^{2+\alpha}+\int_{0}^{t}\left\|\theta_{t}\right\|^{2} d \tau\right)^{7 / 8} .
\end{aligned}
$$


Thus it follows from (2.93) that

$$
\begin{aligned}
\left\|v_{x}\right\|_{L^{\infty}} & \leq C\left(1+\sup _{0 \leq \tau \leq t}\|\theta\|_{L^{\infty}}+\sup _{0 \leq \tau \leq t}\|\zeta\|_{L^{\infty}}\right) \\
& \leq C\left(1+\sup _{0 \leq \tau \leq t}\|\theta\|_{L^{\infty}}+\left(\int_{0}^{t}\left\|\theta_{t}\right\|^{2} d \tau\right)^{7 / 16}\right) .
\end{aligned}
$$

Thus the proof is complete.

After the preparation in the above lemma, we are now in a position to obtain some key estimates.

LEMMA 2.6. For any $t>0$, the following estimates hold:

$$
\begin{gathered}
\left\|\theta_{x}\right\|^{2}+\int_{0}^{t}\left\|\theta_{t}\right\|^{2} d \tau \leq C, \\
\|\theta\|_{L^{\infty}} \leq C, \quad\left\|v_{x}\right\|_{L^{\infty}} \leq C .
\end{gathered}
$$

Proof. Multiplying (1.13) by $\theta_{t}$ and integrating with respect to $x$ yields

$$
\begin{aligned}
\frac{1}{2} \frac{d}{d t}\left\|\theta_{x}\right\|^{2}+\left\|\theta_{t}\right\|^{2} & =\int_{0}^{1} v_{x}^{2} \theta_{t} d x+\int_{0}^{1} f_{1}(u) \theta v_{x} \theta_{t} d x \\
& \leq\left\|v_{x}\right\|_{L^{\infty}}\left\|\theta_{t}\right\|\left\|v_{x}\right\|+C\left\|v_{x}\right\|\left\|\theta_{t}\right\|\|\theta\|_{L^{\infty}}
\end{aligned}
$$

It follows from estimates in previous lemmata that for $\alpha \in(0,1)$ small enough,

$$
\begin{aligned}
& \left|\int_{0}^{t}\|\theta\|_{L^{\infty}}\left\|\theta_{t}\right\|\left\|v_{x}\right\| d \tau\right| \\
& \quad \leq \frac{1}{4} \int_{0}^{t}\left\|\theta_{t}\right\|^{2} d \tau+C \int_{0}^{t}\left\|\theta v_{x}\right\|^{2} d \tau \\
& \quad \leq \frac{1}{4} \int_{0}^{t}\left\|\theta_{t}\right\|^{2} d \tau+C \sup _{0 \leq \tau \leq t}\|\theta\|_{L^{\infty}}^{2} \int_{0}^{t}\left\|v_{x}\right\|^{2} \\
& \quad \leq \frac{1}{4} \int_{0}^{t}\left\|\theta_{t}\right\|^{2} d \tau+C \sup _{0 \leq \tau \leq t}\|\theta\|_{L^{\infty}}^{2+\alpha},
\end{aligned}
$$

and

$$
\begin{aligned}
& \left|\int_{0}^{t}\left\|v_{x}\right\|\left\|\theta_{t}\right\|\left\|v_{x}\right\|_{L^{\infty}} d \tau\right| \\
& \quad \leq C \sup _{0 \leq \tau \leq t}\left\|v_{x}\right\|_{L^{\infty}}\left(\int_{0}^{t}\left\|v_{x}\right\|^{2} d \tau\right)^{1 / 2}\left(\int_{0}^{t}\left\|\theta_{t}\right\|^{2} d \tau\right)^{1 / 2} \\
& \quad \leq C \sup _{0 \leq \tau \leq t}\|\theta\|_{L^{\infty}}^{\alpha / 2}\left(1+\sup _{0 \leq \tau \leq t}\|\theta\|_{L^{\infty}}+\int_{0}^{t}\left\|\theta_{t}\right\|^{2} d \tau\right)^{7 / 16}\left(\int_{0}^{t}\left\|\theta_{t}\right\|^{2} d \tau\right)^{1 / 2} \\
& \quad \leq \frac{1}{4} \int_{0}^{t}\left\|\theta_{t}\right\|^{2} d \tau+C\left(1+\sup _{0 \leq \tau \leq t}\|\theta\|_{L^{\infty}}^{2+\alpha}\right) .
\end{aligned}
$$


Combining (2.107) with (2.108) and (2.109) yields

$$
\left\|\theta_{x}\right\|^{2}+\int_{0}^{t}\left\|\theta_{t}\right\|^{2} d \tau \leq C\left(1+\sup _{0 \leq \tau \leq t}\|\theta\|_{L^{\infty}}^{2+\alpha}\right) .
$$

By Nirenberg's inequality, we have

$$
\|\theta\|_{L^{\infty}} \leq C\left\|\theta_{x}\right\|^{2 / 3}\|\theta\|_{L^{1}}^{1 / 3}+C^{\prime}\|\theta\|_{L^{1}} \leq C\left\|\theta_{x}\right\|^{2 / 3}+C .
$$

Since $\alpha$ can be chosen sufficiently small, from (2.110), (2.111), and Young's inequality it follows that

$$
\left\|\theta_{x}\right\|^{2}+\int_{0}^{t}\left\|\theta_{t}\right\|^{2} d \tau \leq \frac{1}{2} \sup _{0 \leq \tau \leq t}\left\|\theta_{x}\right\|^{2}+C .
$$

Taking the supremum with respect to $t$ in both sides, we get

$$
\sup _{0 \leq \tau \leq t}\left\|\theta_{x}\right\|^{2}+\int_{0}^{t}\left\|\theta_{t}\right\|^{2} \leq C .
$$

Estimates (2.106) follow from Nirenberg's inequality and Lemma 2.5. Thus the proof is complete.

Combining the results obtained in Lemmata 2.2-2.6 and using Eqs. (1.11)-(1.13), we immediately conclude that

LEMma 2.7. For any $t>0$, the following estimates hold:

$$
\begin{gathered}
\left\|v_{x}\right\|^{2}+\int_{0}^{t}\left\|v_{t}\right\|^{2} d \tau+\int_{0}^{t}\left\|v_{x}\right\|^{2} d \tau \leq C, \\
\int_{0}^{t}\left\|\sigma_{1}^{*}\right\|^{2} d \tau \leq C \\
\int_{0}^{t}\left\|\theta_{x}\right\|_{H^{1}}^{2} d \tau \leq C .
\end{gathered}
$$

3. Global existence and uniqueness. In this section we extend an idea by Andrews [2] to prove global existence and uniqueness of weak solutions to the system (1.11)(1.13) with initial data $\left(u_{0}, v_{0}, \theta_{0}\right) \in L^{\infty} \times W_{0}^{1, \infty} \times H^{1}$. The first step is to approximate $u_{0}, v_{0}, \theta_{0}$ by a sequence of smooth functions $u_{0}^{(n)}, v_{0}^{(n)}, \theta_{0}^{(n)}$ such that

$$
\begin{aligned}
& u_{0}^{(n)} \in C^{\infty}, \quad v_{0}^{(n)} \in C_{0}^{\infty}, \quad \theta_{0}^{(n)} \in C^{\infty}, \quad \theta_{0}^{(n)}>0, \quad \forall x \in[0,1], \\
& u_{0}^{(n)} \stackrel{*}{\rightarrow} u_{0} \quad \text { in } L^{\infty}, \quad u_{0}^{(n)} \rightarrow u_{0} \quad \text { in } L^{2}, \\
& v_{0}^{(n)} \stackrel{*}{\rightarrow} v_{0} \quad \text { in } W_{0}^{1, \infty}, \quad v_{0}^{(n)} \rightarrow v_{0} \quad \text { in } H_{0}^{1}, \\
& \theta_{0}^{(n)} \rightarrow \theta_{0} \text { in } H^{1} \text {. }
\end{aligned}
$$

Therefore, there exists a positive constant $M$ depending only on the norm of $u_{0}, v_{0}, \theta_{0}$ in $L^{\infty} \times W^{1, \infty} \times H^{1}$ such that

$$
\left\|u_{0}^{(n)}\right\|_{L^{\infty}}+\left\|v_{0}^{(n)}\right\|_{W_{0}^{1, \infty}}+\left\|\theta_{0}^{(n)}\right\|_{H^{1}} \leq M .
$$


By the results obtained in the previous section and by Chen and Hoffman [4], for the initial data $\left(u_{0}^{(n)}, v_{0}^{(n)}, \theta_{0}^{(n)}\right)$, the following problem

$$
\begin{gathered}
u_{t}-v_{x}=0, \\
v_{t}-v_{x x}-\sigma_{1 x}=0,\left.\quad v\right|_{x=0,1}=0, \\
\theta_{t}-\theta_{x x}-f_{1} \theta v_{x}-v_{x}^{2}=0,\left.\quad \theta_{x}\right|_{x=0,1}=0 \\
t=0: u=u_{0}^{(n)}, v=v_{0}^{(n)}, \theta=\theta_{0}^{(n)}
\end{gathered}
$$

admits a unique global smooth solution $\left(u^{(n)}, v^{(n)}, \theta^{(n)}\right)$. Moreover, for any $t>0$, there exists a positive constant $R$ depending only on the norm of $u_{0}, v_{0}, \theta_{0}$ in $L^{\infty} \times W^{1, \infty} \times H^{1}$, but independent of $t, n$ such that

$$
\begin{aligned}
\left\|u^{(n)}(t)\right\|_{L^{\infty}}+\left\|v^{(n)}(t)\right\|_{W_{0}^{1, \infty}}+\left\|\theta^{(n)}(t)\right\|_{H^{1}} & \leq R, \\
\left\|\theta^{(n)}(t)\right\|_{L^{\infty}}+\left\|\sigma_{1}^{(n)}(t)\right\|_{L^{\infty}} & \leq R, \\
\int_{0}^{t}\left\|v^{(n)}\right\|_{H^{1}}^{2} d \tau+\int_{0}^{t}\left\|v_{t}^{(n)}\right\|^{2} d \tau & \leq R, \\
\int_{0}^{t}\left(\left\|\theta_{x}^{(n)}\right\|_{H^{1}}^{2}+\left\|\theta_{t}^{(n)}\right\|^{2}\right) d \tau & \leq R .
\end{aligned}
$$

We now proceed to investigate convergence of the sequence $\left(u^{(n)}, v^{(n)}, \theta^{(n)}\right)$.

LEMMA 3.1. For any $T>0$, there exists a constant $C_{T}$ such that for all $t \in[0, T]$,

$$
\begin{aligned}
& \left\|u^{(m)}-u^{(n)}\right\|^{2}+\left\|v^{(m)}-v^{(n)}\right\|^{2}+\left\|\theta^{(m)}-\theta^{(n)}\right\|^{2} \\
& \quad \leq C_{T}\left(\left\|u_{0}^{(m)}-u_{0}^{(n)}\right\|^{2}+\left\|v_{0}^{(m)}-v_{0}^{(n)}\right\|^{2}+\left\|\theta_{0}^{(m)}-\theta_{0}^{(n)}\right\|^{2}\right) .
\end{aligned}
$$

REMARK 3.2. From this Lemma one can easily get the uniqueness of weak solutions.

Proof. Multiplying (3.6) satisfied by $u^{(m)}-u^{(n)}$ by $u^{(m)}-u^{(n)}$ and integrating with respect to $x$ yields

$$
\frac{1}{2} \frac{d}{d t}\left\|u^{(m)}-u^{(n)}\right\|^{2} \leq \frac{1}{2}\left\|u^{(m)}-u^{(n)}\right\|^{2}+\frac{1}{2}\left\|v_{x}^{(m)}-v_{x}^{(n)}\right\|^{2} .
$$

Hence,

$$
\left\|u^{(m)}-u^{(n)}\right\|^{2} \leq e^{t}\left\|u_{0}^{(m)}-u_{0}^{(n)}\right\|^{2}+e^{t} \int_{0}^{t} e^{-\tau}\left\|v_{x}^{(m)}-v_{x}^{(n)}\right\|^{2} d \tau
$$

Similarly, from (3.7) it follows that

$$
\frac{1}{2} \frac{d}{d t}\left\|v^{(m)}-v^{(n)}\right\|^{2}+\left\|v_{x}^{(m)}-v_{x}^{(n)}\right\|^{2} \leq \frac{1}{2}\left\|v_{x}^{(m)}-v_{x}^{(n)}\right\|^{2}+C\left\|\sigma_{1}^{(m)}-\sigma_{1}^{(n)}\right\|^{2},
$$

with $\sigma_{1}^{(n)}=f_{1}\left(u^{(n)}\right) \theta^{(n)}+f_{2}\left(u^{(n)}\right)$. Thus we deduce from (3.17) that

$$
\begin{aligned}
\| v^{(m)} & -v^{(n)}\left\|^{2}+\int_{0}^{t}\right\| v_{x}^{(m)}-v_{x}^{(n)} \|^{2} \\
& \leq\left\|v_{0}^{(m)}-v_{0}^{(n)}\right\|^{2}+C \int_{0}^{t}\left(\left\|u^{(m)}-u^{(n)}\right\|^{2}+\left\|\theta^{(m)}-\theta^{(n)}\right\|^{2}\right) d \tau .
\end{aligned}
$$


By the energy method we can deduce from (3.8) that

$$
\begin{aligned}
\| \theta^{(m)}- & \theta^{(n)}\left\|^{2}+\int_{0}^{t}\right\| \theta_{x}^{(m)}-\theta_{x}^{(n)} \|^{2} \\
\leq & C\left\|\theta_{0}^{(m)}-\theta_{0}^{(n)}\right\|^{2}+C \int_{0}^{t}\left(\left\|\theta^{(m)}-\theta^{(n)}\right\|^{2}\right. \\
& \left.+\left\|u^{(m)}-u^{(n)}\right\|^{2}+\left\|v_{x}^{(m)}-v_{x}^{(n)}\right\|^{2}\right) d \tau
\end{aligned}
$$

We infer from (3.19), (3.18), and (3.16) that

$$
\begin{aligned}
\| u^{(m)}- & u^{(n)}\left\|^{2}+\right\| \theta^{(m)}-\theta^{(n)}\left\|^{2}+\right\| v^{(m)}-v^{(n)} \|^{2} \\
\leq & C_{T}\left(\left\|u_{0}^{(m)}-u_{0}^{(n)}\right\|^{2}+\left\|v_{0}^{(m)}-v_{0}^{(n)}\right\|^{2}+\left\|\theta_{0}^{(m)}-\theta_{0}^{(n)}\right\|^{2}\right) \\
& +C_{T} \int_{0}^{t}\left(\left\|u^{(m)}-u^{(n)}\right\|^{2}+\left\|\theta^{(m)}-\theta^{(n)}\right\|^{2}\right) d \tau .
\end{aligned}
$$

By Gronwall's inequality, we obtain (3.14).

From this lemma it follows that $\left(u^{(m)}, v^{(m)}, \theta^{(m)}\right)$ is a Cauchy sequence and there exists $(u, v, \theta) \in C\left([0, T] ; L^{2}\right) \times C\left([0, T] ; L^{2}\right) \times C\left([0, T] ; L^{2}\right)$ such that

$$
\left\|u^{(m)}-u\right\|+\left\|v^{(m)}-v\right\|+\left\|\theta^{(m)}-\theta\right\| \rightarrow 0 \quad \text { as } m \rightarrow \infty .
$$

Combining (3.21) with (3.18) yields that for any $t \in[0, T]$,

$$
\int_{0}^{t}\left\|v_{x}^{(m)}-v_{x}^{(n)}\right\|^{2} d \tau \rightarrow 0 \quad \text { as } m, n \rightarrow \infty .
$$

From (3.22), (3.19) it also follows that

$$
\int_{0}^{t}\left\|\theta_{x}^{(m)}-\theta_{x}^{(n)}\right\|^{2} d \tau \rightarrow 0 \quad \text { as } m, n \rightarrow \infty .
$$

Furthermore, we have

LEMma 3.3. For any $t \in[0, T]$, we have

$$
\begin{gathered}
\left\|v_{x}^{(m)}-v_{x}^{(n)}\right\|^{2}+\left\|\theta_{x}^{(m)}-\theta_{x}^{(n)}\right\|^{2} \rightarrow 0 \quad \text { as } m, n \rightarrow \infty, \\
\int_{0}^{t}\left(\left\|v_{t}^{(m)}-v_{t}^{(n)}\right\|^{2}+\left\|\theta_{t}^{(m)}-\theta_{t}^{(n)}\right\|^{2}\right) d \tau \rightarrow 0 \quad \text { as } m, n \rightarrow \infty .
\end{gathered}
$$

Proof. From (3.7) it easily follows that

$$
\begin{aligned}
0= & \frac{1}{2} \frac{d}{d t}\left\|v_{x}^{(m)}-v_{x}^{(n)}\right\|^{2}+\left\|v_{t}^{(m)}-v_{t}^{(n)}\right\|^{2} \\
& +\frac{d}{d t} \int_{0}^{1}\left(\sigma_{1}^{(m)}-\sigma_{1}^{(n)}\right)\left(v_{x}^{(m)}-v_{x}^{(n)}\right) d x \\
& -\int_{0}^{1}\left(\sigma_{1}^{(m)}-\sigma_{1}^{(n)}\right)_{t}\left(v_{x}^{(m)}-v_{x}^{(n)}\right) d x .
\end{aligned}
$$


Thus, integrating with respect to $t$ yields

$$
\begin{aligned}
\| v_{x}^{(m)}- & v_{x}^{(n)}\left\|^{2}+\int_{0}^{t}\right\| v_{t}^{(m)}-v_{t}^{(n)} \|^{2} d \tau \\
\leq & C\left(\left\|v_{0 x}^{(m)}-v_{0 x}^{(n)}\right\|^{2}+\left\|\left.\left(\sigma_{1}^{(m)}-\sigma_{1}^{(n)}\right)\right|_{t=0}\right\|^{2}+\left\|\left(\sigma_{1}^{(m)}-\sigma_{1}^{(n)}\right)\right\|^{2}\right) \\
& +C \int_{0}^{t}\left(\left\|v_{x}^{(m)}-v_{x}^{(n)}\right\|^{2}+\left\|\theta_{t}^{(m)}-\theta_{t}^{(m)}\right\|^{2}+\left\|u^{(m)}-u^{(n)}\right\|^{2}+\left\|\theta^{(m)}-\theta^{(n)}\right\|^{2}\right) d \tau \\
\leq & C\left(\left\|v_{0 x}^{(m)}-v_{0 x}^{(n)}\right\|^{2}+\left\|u_{0}^{(m)}-u_{0}^{(n)}\right\|^{2}+\left\|\theta_{0}^{(m)}-\theta_{0}^{(n)}\right\|^{2}\right. \\
& \left.+\left\|u^{(m)}-u^{(n)}\right\|^{2}+\left\|\theta^{(m)}-\theta^{(n)}\right\|^{2}\right) \\
& +C \int_{0}^{t}\left(\left\|v_{x}^{(m)}-v_{x}^{(n)}\right\|^{2}+\left\|\theta_{t}^{(m)}-\theta_{t}^{(n)}\right\|^{2}\right. \\
& \left.+\left\|u^{(m)}-u^{(n)}\right\|^{2}+\left\|\theta^{(m)}-\theta^{(n)}\right\|^{2}\right) d \tau .
\end{aligned}
$$

Similarly, we can deduce from (3.8) that

$$
\begin{aligned}
& \frac{1}{2} \frac{d}{d t}\left\|\theta_{x}^{(m)}-\theta_{x}^{(n)}\right\|^{2}+\left\|\theta_{t}^{(m)}-\theta_{t}^{(n)}\right\|^{2} \\
& \quad \leq \frac{1}{2}\left\|\theta_{t}^{(m)}-\theta_{t}^{(n)}\right\|^{2}+C\left(\left\|u^{(m)}-u^{(n)}\right\|^{2}+\left\|\theta^{(m)}-\theta^{(n)}\right\|^{2}+\left\|v_{x}^{(m)}-v_{x}^{(n)}\right\|^{2}\right) .
\end{aligned}
$$

Hence,

$$
\begin{aligned}
\| \theta_{x}^{(m)}- & \theta_{x}^{(n)}\left\|^{2}+\int_{0}^{t}\right\| \theta_{t}^{(m)}-\theta_{t}^{(n)} \|^{2} d \tau \\
\leq & C\left(\left\|\theta_{0 x}^{(m)}-\theta_{0 x}^{(n)}\right\|^{2}+\int_{0}^{t}\left(\left\|u^{(m)}-u^{(n)}\right\|^{2}\right.\right. \\
& \left.\left.+\left\|v_{x}^{(m)}-v_{x}^{(n)}\right\|^{2}+\left\|\theta^{(m)}-\theta^{(n)}\right\|^{2}\right) d \tau\right) \rightarrow 0 \quad \text { as } m, n \rightarrow \infty .
\end{aligned}
$$

Combining this with (3.27) and applying Gronwall's inequality yields that for any $t \in$ $[0, T]$

$$
\left\|v_{x}^{(m)}-v_{x}^{(n)}\right\|^{2}+\int_{0}^{t}\left\|v_{t}^{(m)}-v_{t}^{(n)}\right\|^{2} d \tau \rightarrow 0 \quad \text { as } m, n \rightarrow \infty .
$$

Thus the proof is complete.

We can deduce from (3.30), (3.22), (3.21), and (3.7) that $\forall \varphi \in L^{2}\left([0, T] ; H_{0}^{1}\right)$,

$$
\begin{aligned}
0=\int_{0}^{T} \int_{0}^{1} v_{t}^{(n)} \varphi d x d t+\int_{0}^{T} & \int_{0}^{1}\left(v_{x}^{(n)}+\sigma_{1}^{(n)}\right) \varphi_{x} d x d t \\
& \rightarrow \int_{0}^{T} \int_{0}^{1} v_{t} \varphi d x d t+\int_{0}^{T} \int_{0}^{1}\left(v_{x}+\sigma_{1}\right) \varphi_{x} d x d t
\end{aligned}
$$

Let $w \in L^{2}\left([0, T] ; H^{1}\right)$. Then in the same manner we deduce that the following holds:

$$
\begin{aligned}
0=\int_{0}^{T} \int_{0}^{1}\left(\theta_{t}^{(n)}-f_{1}\left(u^{(n)}\right) \theta^{(n)} v_{x}^{(n)}-\left(v_{x}^{(n)}\right)^{2}\right) w d x d t+\int_{0}^{T} \int_{0}^{1} \theta_{x}^{(n)} w_{x} d x d t \\
\rightarrow \int_{0}^{T} \int_{0}^{1}\left(\theta_{t}-f_{1} \theta v_{x}-v_{x}^{2}\right) w d x d t+\int_{0}^{T} \int_{0}^{1} \theta_{x} w_{x} d x d t
\end{aligned}
$$


It follows from

$$
u^{(n)}(t)=u_{0}^{(n)}+\int_{0}^{t} v_{x}^{(n)} d \tau
$$

and from Lemmas 3.1 and 3.3 that the limit functions $u, v, \theta$ also satisfy Eq. (3.6) in the distribution sense. Therefore, in order to show that the limit functions $u, v, \theta$ are a weak solution to our problem, it remains to prove

Lemma 3.4. For any $T>0$,

$$
u \in C\left([0, T] ; L^{\infty}\right), \quad v \in C\left([0, T] ; W_{0}^{1, \infty}\right), \quad \theta \in C\left([0, T] ; H^{1}\right) .
$$

Proof. By (3.13) we deduce that there is a subsequence of $u^{(n)}, v^{(n)}$ weakly star convergent to $u, v$ in $L^{\infty}\left([0, T] ; L^{\infty}\right) \times L^{\infty}\left([0, T] ; W^{1, \infty}\right)$. Thus we have $u \in L^{\infty}\left([0, T] ; L^{\infty}\right)$, and by (3.6), $u_{t} \in L^{\infty}\left([0, T] ; L^{\infty}\right)$. Thus, $u \in C\left([0, T] ; L^{\infty}\right)$. It easily follows from Lemma 3.1 , Lemma 3.3 that $\theta \in C\left([0, T] ; H^{1}\right)$. It remains to prove that $v \in C\left([0, T] ; W_{0}^{1, \infty}\right)$. By Green's formula, we still have

$$
\begin{aligned}
v_{x}= & \int_{0}^{1} H(x, y, t) v_{0}^{\prime}(y) d y+\int_{0}^{1} H(x, y, t) \sigma_{1}^{*}(y, 0) d y-\sigma_{1}^{*}(x, t) \\
& +\int_{0}^{t} \int_{0}^{1} H(x, y, t-s) \sigma_{1 s}^{*}(y, s) d y d s .
\end{aligned}
$$

It turns out from the smooth property of the heat equation that the first three terms on the right-hand side of $(3.34)$ belong to $C\left([0, T] ; L^{\infty}\right)$. The last term on the righthand side satisfies $(2.95)-(2.96)$. Since $\sigma_{1 t}^{*}$ belongs to $L^{2}\left([0, T] ; L^{2}\right)$, from the standard result in parabolic equations it follows that the last term belongs to $C\left([0, T] ; H^{1}\right) \subset$ $C\left([0, T] ; L^{\infty}\right)(n=1)$. Thus the proof is complete.

REMARK 3.5. Since all constants in all uniform a priori estimates in section 2 depend only on the $L^{\infty} \times W^{1, \infty} \times H^{1}$ norm of $\left(u_{0}, v_{0}, \theta_{0}\right)$, it is easy to see that all estimates in section 2 still hold for weak solutions.

4. Asymptotic behavior. In this section we shall prove the results on asymptotic behavior of solutions given in Theorem 1.2. A convergence symbol " $\rightarrow$ " is to be understood as $t \rightarrow \infty$. We shall make use of the following basic lemma from Shen and Zheng [17]:

Lemma 4.1 ([17]). Suppose $y$ and $h$ are nonnegative functions on $[0, \infty), y^{\prime}$ is locally integrable, and $y, h$ satisfy

$$
\begin{array}{ll}
\forall t \geq 0: & y^{\prime}(t) \leq A_{1} y^{2}(t)+A_{2}+h(t), \\
\forall T>0: \quad \int_{0}^{T} y(\tau) d \tau \leq A_{3}, \quad \int_{0}^{T} h(\tau) d \tau \leq A_{4},
\end{array}
$$

with $A_{1}, A_{2}, A_{3}, A_{4}$ being positive constants independent of $t$ and $T$. Then for any $r>0$

$$
\forall t \geq 0: \quad y(t+r) \leq\left(\frac{A_{3}}{r}+A_{2} r+A_{4}\right) e^{A_{1} A_{2}} .
$$


Moreover,

$$
\lim _{t \rightarrow \infty} y(t)=0
$$

LEMMA 4.2.

$$
\begin{gathered}
\|v(t)\|_{H_{0}^{1}} \rightarrow 0, \\
\left\|\sigma_{1}^{*}\right\| \rightarrow 0, \quad\left\|\sigma^{*}\right\| \rightarrow 0 .
\end{gathered}
$$

Proof. Multiplying (2.38) by $-\int_{0}^{x} \sigma_{1 t}^{*} d x$ and integrating with respect to $x$ yields

$$
\begin{aligned}
\frac{1}{2} \frac{d}{d t}\left\|\sigma_{1}^{*}\right\|^{2} & =\left(v_{t},-\int_{0}^{x} \sigma_{1 t}^{*} d x\right)+\left(-v_{x}, \sigma_{1 t}^{*}\right) \\
& \leq C\left(\left\|v_{t}\right\|^{2}+\left\|v_{x}\right\|^{2}+\left\|\theta_{t}\right\|^{2}\right) .
\end{aligned}
$$

From Lemmata 4.1, 2.7, and 2.6 it follows that

$$
\left\|\sigma_{1}^{*}\right\|^{2} \rightarrow 0
$$

To prove $\left\|v_{x}\right\| \rightarrow 0$, we take the expression (3.35) of $v_{x}$ into consideration. It is easy to see that the first two terms on the right-hand side converge to zero in $L^{2}$ as time tends to infinity. By (4.8), the third term also converges to zero in $L^{2}$. Let the last term be $\zeta$. Then $\zeta$ satisfies $(2.95)-(2.96)$. We easily deduce that

$$
\begin{aligned}
\frac{1}{2} \frac{d}{d t}\|\zeta\|^{2}+\left\|\zeta_{x}\right\|^{2} & \leq \varepsilon\|\zeta\|^{2}+\left\|\sigma_{1 t}^{*}\right\|^{2} \\
& \leq C \varepsilon\left\|\zeta_{x}\right\|^{2}+C\left(\left\|v_{x}\right\|^{2}+\left\|\theta_{t}\right\|^{2}\right) .
\end{aligned}
$$

Choosing $\varepsilon$ small enough and applying Lemma 2.6, Lemma 2.7, we get

$$
\int_{0}^{t}\|\zeta\|^{2} d \tau \leq C \int_{0}^{t}\left\|\zeta_{x}\right\|^{2} d \tau \leq C .
$$

Combining (4.9) with (4.10) and applying Lemma 4.1 yields (4.5). The second one in (4.6) follows from the first one and (4.5). Thus the proof is complete.

LEMMA 4.3.

$$
\begin{aligned}
\left\|\theta_{x}(t)\right\| & \rightarrow 0 \\
\|\theta(t)-\bar{\theta}(t)\|_{L^{\infty}} & \rightarrow 0
\end{aligned}
$$

Proof. Since

$$
\begin{aligned}
\frac{1}{2} \frac{d}{d t}\left\|\theta_{x}\right\|^{2}+\left\|\theta_{t}\right\|^{2} & =\int_{0}^{1} v_{x}^{2} \theta_{t} d x+\int_{0}^{1} f_{1}(u) \theta \theta_{t} v_{x} d x \\
& \leq \frac{1}{2}\left\|\theta_{t}\right\|^{2}+\left\|v_{x}\right\|_{L^{\infty}}^{2}\left\|v_{x}\right\|^{2}+C\left\|v_{x}\right\|^{2}
\end{aligned}
$$

Lemmata 2.6, 2.7, and 4.1 yield (4.11). By the Poincaré inequality and (4.11), we conclude that

$$
\|\theta(t, \cdot)-\bar{\theta}(t)\|_{L^{\infty}} \leq C\left\|\theta_{x}(t)\right\| \rightarrow 0
$$

Thus the proof is complete. 


\section{REFERENCES}

[1] H. W. Alt, K. H. Hoffmann, M. Niezgódka, and J. Sprekels, A numerical study of structural phase transitions in shape memory alloys, Inst. Math. Univ. Augsburg, preprint No. 90, 1985

[2] G. Andrews, On the existence of solutions to the equation $u_{t t}=u_{x x t}+\sigma\left(u_{x}\right)_{x}$, J. Differential Equations 35, 200-231 (1980)

[3] G. Andrews and J. M. Ball, Asymptotic behaviour and changes of phase in one-dimensional nonlinear viscoelasticity, J. Differential Equations 44, 306-341 (1982)

[4] Z. Chen and K. H. Hoffmann, On a one-dimensional nonlinear thermoviscoelastic model for structural phase transitions in shape memory alloys, J. Differential Equations 112, 325-350 (1994)

[5] C. M. Dafermos, Global smooth solutions to the initial boundary value problem for the equations of one-dimensional nonlinear thermoviscoelasticity, SIAM J. Math. Anal. 13, 397-408 (1982)

[6] C. M. Dafermos and L. Hsiao, Global smooth thermomechanical processes in one-dimensional nonlinear thermoviscoelasticity, Nonlinear Analysis 6, 435-454 (1982)

[7] F. Falk, Ginzburg-Laudau theory of static domain walls in shape memory alloys, Physica B 51, 177-185 (1983)

[8] F. Falk, Ginzburg-Laudau theory and solitary waves in shape memory alloys, Physica B 54, 159-167 (1984)

[9] K. H. Hoffmann and S. Zheng, Uniqueness for structural phase transitions in shape memory alloys, Math. Meth. Appl. Sci. 10, 145-151 (1988)

[10] K. H. Hoffmann and A. Zochowski, Existence of solutions to some non-linear thermoelastic systems with viscosity, Math. Meth. Appl. Sci. 15, 187-204 (1992)

[11] S. Jiang, Global large solutions to initial boundary value problems in one-dimensional nonlinear thermoviscoelasticity, Quart. Appl. Math. 51, 731-744 (1993)

[12] T. Luo, Qualitative behavior to nonlinear evolution equations with dissipation, Ph.D. Thesis, Institute of Mathematics, Academy of Sciences of China, Bejing, 1994

[13] M. Niezgódka and J. Sprekels, Existence of solutions for a mathematical model of structural phase transitions in shape memory alloys, Math. Meth. Appl. Sci. 10, 197-223 (1988)

[14] M. Niezgódka, S. Zheng, and J. Sprekels, Global solutions to a model of structural phase transitions in shape memory alloys, J. Math. Anal. Appl. 130, 39-54 (1988)

[15] R. L. Pego, Phase transitions in one-dimensional nonlinear viscoelasticity: admissibility and stability, Arch. Rational Mech. Anal. 97, 353-394 (1987)

[16] R. Racke and S. Zheng, Global existence and asymptotic behavior in nonlinear thermoviscoelasticity, J. Differential Equations 134, 46-67 (1997)

[17] W. Shen and S. Zheng, On the coupled Cahn-Hilliard equations, Comm. Partial Differential Equations 18, 701-727 (1993)

[18] J. Sprekels and S. Zheng, Global solutions to the equations of a Ginzburg-Landau theory for structural phase transitions in shape memory alloys, Physica D 39, 59-76 (1989)

[19] J. Sprekels, S. Zheng, and P. Zhu, Asymptotic behavior of the solutions to a Laudau-Ginzburg system with viscosity for martensitic phase transitions in shape memory alloys, SIAM J. Math. Anal. 29, No. 1, 69-84 (1998) 